\title{
Mathematical model of the effect of ischemia-reperfusion on brain capillary collapse and tissue swelling
}

\author{
M. J. Mohamed Mokhtarudin ${ }^{\mathrm{a}, \mathrm{b}, *}$, S. J. Payne ${ }^{\mathrm{a}}$ \\ ${ }^{a}$ Institute of Biomedical Engineering, Department of Engineering Science, Old Road Campus Research Building, University \\ of Oxford, Headington, Oxford OX3 $7 D Q, U K$ \\ ${ }^{b}$ Faculty of Mechanical Engineering, University Malaysia Pahang, 26600 Pekan, Pahang, Malaysia
}

\begin{abstract}
Restoration of an adequate cerebral blood supply after an ischemic attack is a primary clinical goal. However, the blood-brain barrier may break down after a prolonged ischemia causing the fluid in the blood plasma to filtrate and accumulate into the cerebral tissue interstitial space. Accumulation of this filtration fluid causes the cerebral tissue to swell, a condition known as vasogenic oedema. Tissue swelling causes the cerebral microvessels to be compressed, which may further obstruct the blood flow into the tissue, thus leading to the no-reflow phenomenon or a secondary ischemic stroke. The actual mechanism of this however is still not fully understood. A new model is developed here to study the effect of reperfusion on the formation of vasogenic oedema and cerebral microvessel collapse. The formation of vasogenic oedema is modelled using the capillary filtration equation while vessel collapse is modelled using the tube law of microvessel. Tissue swelling is quantified in terms of displacement, which is modelled using poroelastic theory. The results show that there is an increase in tissue displacement and interstitial pressure after reperfusion. In addition, the results also show that vessel collapse can occur at high value of reperfusion pressure, high blood osmotic pressure, high cerebral capillary permeability and low cerebral capillary stiffness. This model provides insight on the formation on the formation of ischemia-reperfusion injury by tissue swelling and vessel collapse.
\end{abstract}

Keywords: Blood-brain barrier, ischemia-reperfusion, cerebral blood flow, vasogenic oedema

\section{Introduction}

In the past decade, stroke has become the second major cause of death in the world after ischaemic heart disease [1. There are two types of stroke; haemorrhagic and ischaemic. Haemorrhagic stroke occurs when a blood vessel ruptures and bleeding occurs in the brain, meanwhile, ischaemic stroke is usually caused by the blockage of a blood vessel in the brain. Stroke can result in various complications such as physical disabilities, problems with vision, problems with speech and understanding, and as well as psychological problems. Stroke incidence rates in developing countries doubled between 1970 and 2008, while the rates have dropped in developed countries by about $42 \%$ 2. Despite this, stroke remains a primary cause of mortality

\footnotetext{
${ }^{*}$ Corresponding author

Email address: mohd.mohamedmokhtarudin@wolfson.ox.ac.uk (M. J. Mohamed Mokhtarudin)
} 
and most patients that survive death suffer from permanent disability and more than $50 \%$ of survivors in the UK live dependently on others for their daily activities [3]. Ischaemic stroke as it contributes to $85 \%$ of stroke incidents and will then be the focus of this study.

About $70 \%$ of patients diagnosed with ischemic stroke have a thrombus blocking a major brain vessel 4. Restoration of the cerebral blood supply within a short timescale is the main goal for clinicians and this process is called reperfusion therapy. Current reperfusion methods aim to remove the blood clot by catheter insertion in the affected vessels [5] or by using recombinant tissue plasminogen activator (rtPA) [6], which aims to break up the blood clot. Although reperfusion is important in restoring the blood supply to the ischemic tissue, it has associated risks and rTPA is only effective within 3 to 4.5 hours from stroke occurrence [7]. Late restoration of blood flow to the affected brain tissue after ischemia may also cause what is known as the no-reflow phenomenon [8] or secondary stroke occurrence. In a systematic meta-analysis study done by [9], the risk of secondary stroke occurrence is the highest in the first month after the first stroke occurrence. This study shows that stroke can reoccur but the mechanism of which it can happen is still not well understood. One hypothesis that tries to explain its occurrence is vessel compression due to the formation of vasogenic oedema after ischemia-reperfusion.

During ischemic stroke, there is a reduction in CBF that causes oxygen deprivation to the brain tissue and that creates a series of molecular processes that produce toxic proteases and free radicals, which are involved in damaging the brain cells and usually lead to the breakdown of blood-brain barrier (BBB) [10]. The BBB is a special barrier that makes the brain capillary vessels different from other capillary vessels found in peripheral organs. The BBB structure is formed by the capillary endothelial cells, which are bounded together by tight junctions. The presence of a tight junction between the cerebral endothelial cells limits the movement of molecules and ions through the capillary [11. The astrocyte foot ends that surround the capillary help in maintaining BBB properties. Meanwhile, the presence of pericytes surrounding the endothelial cells are thought possibly to be responsible for sustaining the BBB permeability, regulating blood flow and helping in the formation of new vessels [12].

Breakdown of the BBB will increase its permeability, allowing the filtration of many molecules and ions from the reperfused blood such as plasma proteins, salts and water [13. Accumulation of this filtration fluid in the tissue interstitial space creates a condition called vasogenic oedema [14, which will increase intracranial pressure (ICP) and cause tissue swelling [15]. These might lead to vessel compression [16], which may obstruct blood flow to a specific region in the brain leading to secondary ischemia or the no-reflow phenomenon after reperfusion. However, this phenomenon remains very poorly understood and so the focus of this study is to investigate using a mathematical model, the no-reflow phenomenon caused by cerebral capillary filtration after BBB breakdown. By doing so, it will be possible to quantify this phenomenon and to explore whether or not it plays an important role in ischemic stroke. 


\section{Theory}

Consider a very small volume of cerebral tissue, which contains a distribution of capillary vessels with different sizes and mechanical properties. In this model, it is assumed that water filtrates from the blood vessels into the cerebral interstitial space through the capillary wall, as shown in Figure 1. After stroke occurrence, it is assumed that the BBB tight junctions start to break and hence the permeability of the wall increases. When blood flows through the cerebral capillaries after reperfusion, filtration of ions and protein plasma from the blood into the tissue interstitial space can occur. This filtration creates an osmotic pressure difference between the interstitial space and the capillary, which causes water flux into the tissue space. Accumulation of water inside the interstitial space can cause tissue displacement. The question to be answered here is whether the resulting pressure difference between the interstitial space and the capillary can cause vessel collapse.

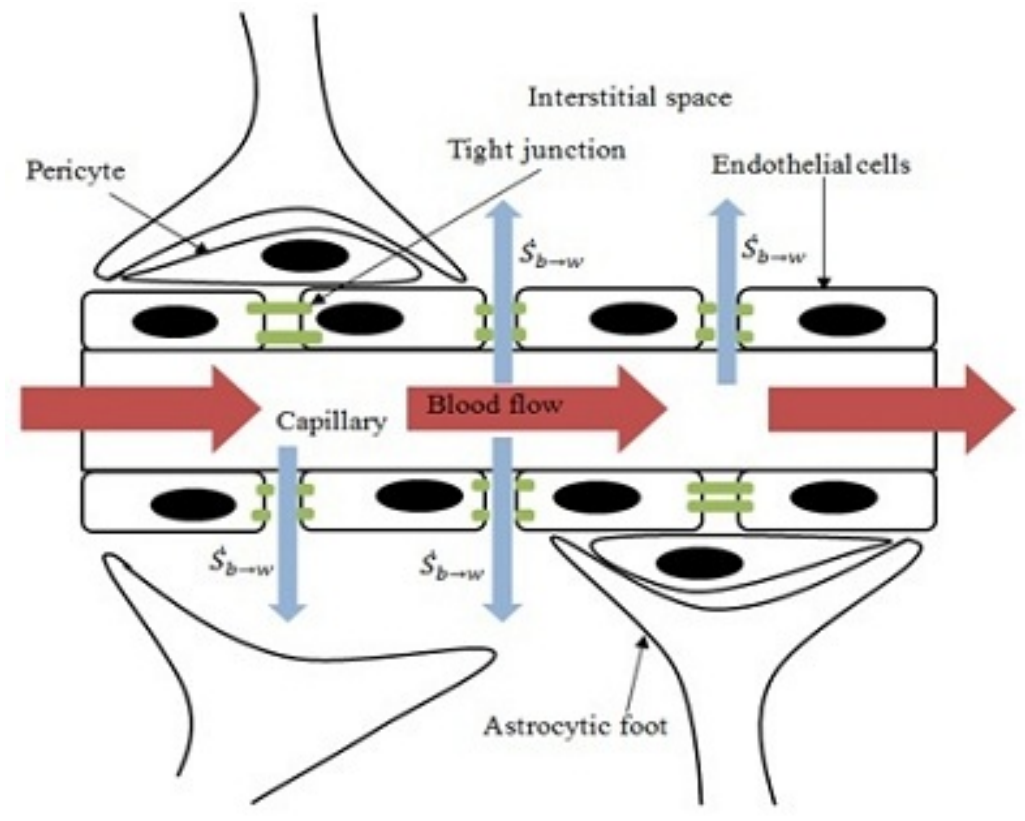

Figure 1: Capillary filtration after BBB breakdown, showing water filtration flux through the endothelium of the capillary with damaged tight junctions.

The capillaries are assumed to be distributed homogeneously inside the cerebral tissue and the permeability of the normal BBB is assumed to be approximately zero, to simplify the proposed mathematical model. The brain tissue is assumed to be elastic, homogeneous and isotropic, and the model treats white and grey matter as mechanically the same.

\subsection{Capillary filtration}

It is assumed that water filtration, $\dot{S}_{b \rightarrow w}$ occurs across the surface area of each vessel within a small volume of cerebral tissue and that this can be approximated by an integration of water flux, $J_{V A}$, at each 
point in space over the distribution of vessel circumferences, $p(c)$, for each vessel of length $L_{c}$, in a given volume as shown below:

$$
\dot{S}_{b \rightarrow w}=\frac{d N}{d V} \int_{0}^{\infty} J_{V A} L_{c} p(c) d c
$$

where the subscripts $b$ and $w$ represent the blood and water phases respectively. The term $\frac{d N}{d V}$ is a scaling factor that represents the number of vessels $d N$ in a given volume $d V$. The baseline volume fraction of the blood is:

$$
\bar{n}_{b}=\frac{d N}{d V} \pi R_{c}^{2} L_{c}
$$

where $R_{c}$ is the baseline value of vessel radius. The term $J_{V A}$ is independent of vessel size and can thus be taken out from the integral. Substituting eqn. 2 into 1 gives:

$$
\dot{S}_{b \rightarrow w}=J_{V A} \frac{\bar{n}_{b}}{\pi R_{c}{ }^{2}} \int_{0}^{\infty} p(c) d c .
$$

The integral above can be simplified by assuming that the vessel perimeter does not change as the wall deforms, hence:

$$
\int_{0}^{\infty} p(c) d c=2 \pi R_{c} f
$$

where $f$ represents the fraction of vessels that remain open at each point because it is assumed that water flux occurs only whilst the vessel is still opens. Thus, eqn. 3 reduces to:

$$
\dot{S}_{b \rightarrow w}=J_{V A} \frac{2 \bar{n}_{b}}{R_{c}} f .
$$

The water filtration flux from pathological brain capillaries into the interstitial space can be described by the Starling principle of capillary filtration, which states that the fluid movement across the capillary wall depends on the difference between the hydrostatic and osmotic pressures between the blood and interstitial fluid (ISF) 17. Hydrostatic pressure is the pressure that is exerted by a fluid at rest, while the osmotic pressure is the pressure exerted by the difference between the concentrations of solutes present in the fluid. The filtration flux, $J_{V A}$, can thus be described by:

$$
J_{V A}=L_{p}\left[\left(P_{b}-P_{w}\right)-\sum_{m=1}^{M}\left(\sigma\left(\Pi_{b}-\Pi_{w}\right)\right)_{m}\right],
$$

where $L_{p}$ is the hydraulic permeability of the capillary wall, $P$ is the hydrostatic pressure and $\Pi$ is the osmotic pressure for each water soluble solute, $m$, present in the blood plasma and ISF. The term is the reflection coefficient, which is in the range of 0 to 1 , with $\sigma=1$ meaning that no solute can pass through the capillary wall. 
In steady state, the ISF protein concentration, $C_{w}$, is not a fixed value but dynamically varying and can be related to the solute flux, $J_{S}$, and water flux through $C_{w}=J_{S} J_{V A}$ [18. The blood plasma protein concentration, together with the definition of $C_{w}$, can be used to express $\Pi_{w}$ in terms of $\Pi_{b}$ as given by eqn. 7 below:

$$
\Pi_{b}-\Pi_{w}=\sigma \Pi_{b} \frac{1-e^{-P e}}{1-\sigma e^{-P e}}
$$

where:

$$
P e=\frac{J_{V A}(1-\sigma) h}{D}
$$

is the Péclet number with $h$ and $D$ denoting the wall thickness and diffusion coefficient respectively. By substituting eqn. 7 into eqn. 6, the filtration flux can be found to be:

$$
J_{V A}=L_{p}\left[\left(P_{b}-P_{w}\right)-\sum_{m=1}^{M}\left(\sigma^{2} \Pi_{b} \frac{1-e^{P e}}{1-\sigma e^{P e}}\right)_{m}\right],
$$

In a normal capillary wall, there exist both small and large pores which allow the flow of solutes such as ions and proteins through the wall [19]. Equation 9 can be extended by adding filtration terms for both small and large pores as shown below:

$$
J_{V A}=\sum_{l=1}^{L} L_{p}\left[\left(P_{b}-P_{w}\right)-\sum_{m=1}^{M}\left(\sigma^{2} \Pi_{b} \frac{1-e^{P e}}{1-\sigma e^{P e}}\right)_{l, m}\right],
$$

wherel represents the different pore sizes present on the wall. In the experiment done by [20] in the rat $\mathrm{BBB}$ after ischemia, it was suggested that there are large pores present in the BBB. The filtration equation can be modified to model the filtration behaviour of a pathological BBB by giving appropriate value of the reflection coefficients of proteins and ions, which can be assumed to be 0.93 and 0 respectively for both pore sizes [21]. Thus, eqn. 10 becomes:

$$
\begin{aligned}
J_{V A} & =L_{p_{S}}\left[\left(P_{b}-P_{w}\right)-\left(\sigma^{2} \Pi_{b} \frac{1-e^{P e}}{1-\sigma e^{P e}}\right)_{\text {protein }, S}\right] \\
& +L_{p_{L}}\left[\left(P_{b}-P_{w}\right)-\left(\sigma^{2} \Pi_{b} \frac{1-e^{P e}}{1-\sigma e^{P e}}\right)_{\text {protein }, L}\right],
\end{aligned}
$$

The subscript L and S represent the large and small pores present in breakdown BBB. Since reflection coefficients for both pores are assumed to be the same [21, the equation can be simplified to:

$$
J_{V A}=L_{p}\left[\left(P_{b}-P_{w}\right)-\left(\sigma^{2} \Pi_{b} \frac{1-e^{P e}}{1-\sigma e^{P e}}\right)\right]
$$

where $L_{p}$ is the combined hydraulic permeability for small and large pores. The value of $L_{p}$ for normal BBB is estimated to be about $2.0 \times 10^{-9} \mathrm{~cm} / \mathrm{s} . \mathrm{cmH} 2 \mathrm{O}[22$ and during ischemia, the value can increase more than 
100 times the normal value [23. This equation is highly nonlinear and can be further simplified by assuming $P e \ll 1$, as will be justified later, and taking the first order approximation using Taylor series expansion to yield:

$$
J_{V A}=L_{p}\left[\left(P_{b}-P_{w}\right)-\sigma \Pi_{b}\right],
$$

Substituting eqn. 13 into 5 gives the final capillary filtration model used here:

$$
\dot{S}_{b \rightarrow w}=2 \bar{n}_{b} \frac{L_{p}}{R_{c}} f\left[\left(P_{b}-P_{w}\right)-\sigma \Pi_{b}\right]
$$

\subsection{Vessel wall model}

Vessels are assumed to collapse when there is a transmural pressure difference and to occur without any change in the perimeter. The transmural pressure can be related to the change in the cross-sectional area of the vessel as given by [24]:

$$
P_{b}-P_{w}=E\left(\frac{A_{i}}{A_{o}}\right)^{k}-P_{c}
$$

where $E$ is the capillary wall stiffness, $A_{i}$ and $A_{o}$ are the deformed and baseline cross-sectional areas respectively, $P_{c}$ is the critical pressure that causes collapse, and $k$ is an exponent. At baseline conditions, $P_{b}-\bar{P}=E-P_{c}$, hence eqn. 15 can be rewritten as:

$$
\frac{A_{i}}{A_{o}}=\left(1+\frac{\bar{P}-P_{w}}{E}\right)^{1 / k}
$$

\subsection{Tissue displacement}

When a pressure gradient develops due to water filtration, the interstitial space expands and tissue will deform. The deformation depends on the tissue elastic properties. One of the most common approaches to study brain tissue deformation is the use of poroelastic theory, which is suitable for modelling long term fluid movement processes [25]. This theory has been used in many similar studies such as those for hydrocephalus [26, 27, 28, neurosurgery [29, 30], brain chemotherapeutic infusion [24], and many others. In this study we model the brain tissue by assuming the brain tissue as a homogeneous and linearly elastic material containing water and blood in a porous structure.

Figure 2 shows a block of tissue containing two different porosities containing fluids A and B, and a solid matrix. The porosity is defined as the volume of a phase divided by the total volume of the system:

$$
n^{a}=\frac{d V^{a}}{d V}
$$

where the superscript $a=1, A$ represents the different fluid networks with different values of porosity present in the system [31, which in the case of Figure 2, equate to fluids A and B. The solid matrix and fluid 
phase displacements can be denoted as $u_{i}$ and $u_{i}^{a}$ respectively. In this theory, the relative fluid displacement is given as [32]:

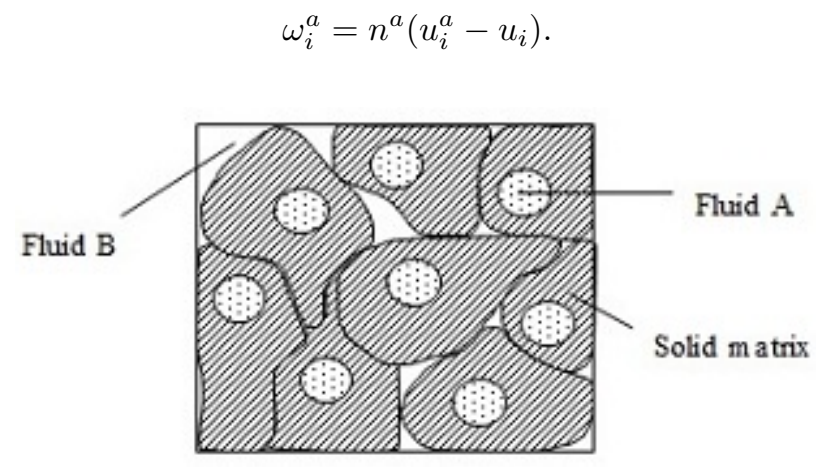

Figure 2: An example of a block with two porosities present.

Terzaghi's principle states that when a porous medium is subjected to a stress, it is opposed by the pressure of each fluid network present [33] as given by:

$$
\sigma_{i j}=\sigma_{i j}^{\prime}-\sum_{a=1}^{A} \alpha^{a} P^{a} \delta_{i j}
$$

where $\sigma_{i j}$ and $\sigma_{i j}^{\prime}$ are the total and effective stresses in the solid matrix respectively, $\alpha$ is the Biot parameter [34, $P$ is the fluid pressure and $\delta_{i j}$ represents the Kronecker delta. In this analysis, the subscripts $i$ and $j$ have values of 1,2 and 3 . For an isotropic and linearly elastic material, the effective stress can be related to the strain by:

$$
\sigma_{i j}^{\prime}=2 G\left(\varepsilon_{i j}+\frac{v}{1-2 v} \varepsilon_{i i}\right),
$$

where $G$ is the shear modulus and $v$ is the Poisson's ratio. The strain-displacement relationship is given by:

$$
\varepsilon_{i j}=\frac{1}{2}\left(u_{i, j}+u_{j, i}\right)
$$

In equilibrium of momentum, the divergence of eqn. 19 equals zero. However, in the presence of local body forces such as gravity and accounting for fluid accelerations, the divergence of the total stress can be related by:

$$
\sigma_{i j, j}+\rho\left(b_{i}-\ddot{u}_{i}\right)=\sum_{a=1}^{A} \rho^{a}\left(\ddot{\omega}_{i}^{a}+\dot{\omega}_{j}^{a} \dot{\omega}_{i, j}^{a}\right),
$$

where $\rho=\sum_{a=1}^{A} n^{a} \rho^{a}+(1-n) \rho^{s}$ is the total density of the system, $\left.n=\sum_{(} a=1\right)^{A} n^{a}$ is the total volume fraction of the combined fluid networks, $\rho^{s}$ is the density of the solid phase, and $b_{i}$ are the local body forces. The solid displacement is described in the Lagrangian frame of reference while the fluid velocities are 
described in the Eulerian frame of reference relative to the solid matrix [35, 36. By substituting eqns. 19 , 20 and 21 into eqn. 22 . the equation for solid phase can be expressed in Del $(\nabla)$ notation as:

$$
G \nabla^{2} u_{i}+\frac{G}{1-2 v} \nabla\left(\nabla \cdot u_{i}\right)-\sum_{a=1}^{A} \alpha^{a} \nabla P^{a}-\sum_{a=1}^{A} \rho^{a}\left(\ddot{\omega}_{i}^{a}+\dot{\omega}_{j}^{a} \cdot \nabla \dot{\omega}_{i, j}^{a}\right)+\rho\left(b_{i}-\ddot{u}_{i}\right)=0 .
$$

For the fluid networks, the fluid flow of fluid phase a can be described by mass conservation [36]:

$$
\frac{1}{Q^{a}} \dot{P}^{a}+\alpha^{a} \dot{\varepsilon}_{i i}+\dot{\omega}_{i, i}^{a}-\sum_{a, b=1, b \neq a}^{A} \dot{S}_{b \rightarrow a}=-\frac{\dot{\rho}}{\rho} \omega_{i}^{a},
$$

where $S_{b \rightarrow a}$ represents the rate of fluid transfer from fluid network $b$ to fluid network $a$. The term $Q^{a}$ is known as the relative compressibility of the fluid network and can be expressed as:

$$
\frac{1}{Q^{a}}=\frac{\alpha^{a}-n^{a}}{K^{S}}+\frac{n^{a}}{K^{a}}
$$

where $K^{s}$ and $K^{a}$ are the bulk moduli for the solid matrix and fluid network respectively. In addition, each fluid network can also be described using equilibrium of momentum, which is given by the sum of the divergence of the fluid pressure, the viscous drag force $R^{a}$, and the external forces and is equal to the acceleration of the fluid network relative to the solid matrix as shown below:

$$
\rho\left(b_{i}-\ddot{u}_{i}\right)-P_{, i}^{a}-R_{i}^{a}=\frac{\rho^{a}}{n^{a}}\left(\ddot{\omega}_{i}^{a}+\dot{\omega}_{j}^{a} \dot{\omega}_{i, j}^{a}\right) .
$$

The term $R^{a}$ can be described by Darcy's Law, which is given by the eqn. 27 below:

$$
R_{i}^{a}=\frac{\dot{\omega}_{i}^{a}}{k^{a}}
$$

where the term $k^{a}$ is known as the permeability of the fluid network, which is usually assumed to be isotropic and which can then be represented by a single value [36]. Substituting eqn. 27 into 26 , rearranging and substituting into eqn. 24 and expressing in $\nabla$ notation as:

$$
\begin{aligned}
\frac{1}{Q^{a}} \dot{P}^{a} & +\alpha^{a} \nabla \cdot \dot{u}_{i}+k^{a} \rho^{a} \nabla \cdot\left(b_{i}-\ddot{u}_{i}\right)-k^{a} \nabla^{2} P^{a} \\
& -\frac{k^{a} \rho^{a}}{n^{a}} \nabla \cdot\left(\ddot{\omega}_{i}^{a}+\dot{\omega}_{j}^{a} \dot{\omega}_{i, j}^{a}\right)-\sum_{a, b=1, b \neq a}^{A} \dot{S}_{b \rightarrow a}+\frac{\dot{\rho}}{\rho} \omega_{i}^{a}=0 .
\end{aligned}
$$

Equations 23 and 28 form the set of equations that can be used to solve for the fluid pressure and solid displacement of the system. However, these equations are highly nonlinear. In biological flows such as water filtration into the cerebral tissue, the fluid acceleration frequencies are low and can be neglected [26]. Further, the fluid densities are assumed constant throughout the process. It is also assumed that the tissue displacement is a slow process so that the time derivative of the solid phase displacement can be neglected. 
The effect of external forces on the system is very small and can be neglected. For the one-dimensional case and assuming spherical symmetry, the equations can thus be simplified to:

$$
\begin{gathered}
G \nabla^{2} u+\frac{G}{1-2 v} \nabla(\nabla \cdot u)-\sum_{a=1}^{A} \alpha^{a} \nabla P^{a}-=0, \\
\frac{1}{Q^{a}} \dot{P}^{a}-k^{a} \nabla^{2} P^{a}-\sum_{a, b=1, b \neq a}^{A} S \cdot b \rightarrow a=0 .
\end{gathered}
$$

In the model proposed here, three phases are involved which are the solid brain tissue phase and two fluid phases, blood and water. Thus, the system of equations can be written out fully as:

$$
\begin{gathered}
\nabla^{2} u-\frac{2}{r^{2}} u=\frac{1-2 v}{2 G(1-v)}\left(\alpha_{b} \frac{\partial P_{b}}{\partial r}+\alpha_{w} \frac{\partial P_{w}}{\partial r}\right), \\
\frac{1}{Q_{b}} \frac{\partial P_{b}}{\partial t}-k_{b} \nabla^{2} P_{b}+\dot{S}_{b \rightarrow w}=0 \\
\frac{1}{Q_{w}} \frac{\partial P_{w}}{\partial t}-k_{w} \nabla^{2} P_{w}-\dot{S}_{b \rightarrow w}=0
\end{gathered}
$$

A positive value of $\dot{S}_{b \rightarrow w}$ represents the direction of the movement of water from the blood capillaries into the interstitial space, while a negative value represents the reverse direction.

\subsection{Non-dimensionalisation}

The system of equations above can be further simplified through the use of non-dimensionalisation. Assuming that the characteristic lengths for the tissue displacement $u$ and distance $r$ are $L_{u}$ and $L$ respectively, the baseline value for all the pressures is $\bar{P}$, and the characteristic time is $T$, the equations can be non-dimensionalised as follows:

$$
\begin{gathered}
\tilde{\nabla}^{2} \tilde{u}-\frac{2}{\tilde{r}^{2}} \tilde{u}=\frac{L \bar{P}(1-2 v)}{2 L_{u} G(1-v)}\left(\alpha_{b} \frac{\partial \tilde{P}_{b}}{\partial \tilde{r}}+\alpha_{w} \frac{\partial \tilde{P}_{w}}{\partial \tilde{r}}\right), \\
\frac{L^{2}}{Q_{b} k_{b} T} \frac{\partial \tilde{P}_{b}}{\partial \tilde{t}}-k_{b} \tilde{\nabla}^{2} \tilde{P}_{b}+2 \bar{n}_{b}\left(\frac{L_{p} L^{2}}{R_{c} k_{b}}\right) f\left[\left(\tilde{P}_{b}-\tilde{P}_{w}\right)-\sigma \tilde{\Pi}_{b}\right]=0, \\
\frac{L^{2}}{Q_{w} k_{w} T} \frac{\partial \tilde{P}_{w}}{\partial \tilde{t}}-k_{w} \tilde{\nabla}^{2} \tilde{P}_{w}-2 \bar{n}_{b}\left(\frac{L_{p} L^{2}}{R_{c} k_{w}}\right) f\left[\left(\tilde{P}_{b}-\tilde{P}_{w}\right)-\sigma \tilde{\Pi}_{b}\right]=0,
\end{gathered}
$$

where the tilde $(\sim)$ symbol is used to represent the non-dimensionalised variables. Without loss of generality, by setting the coefficient on the right hand side of eqn. 34 equal to one, the relationship between the two characteristic lengths becomes:

$$
L_{u}=\frac{\bar{P}(1-2 v)}{2 G(1-v)} L
$$


Coefficient in the round bracket of eqn. 36 is set equal to one to determine a suitable characteristic length. Using the parameter values given in Table 1 the characteristic length chosen here is:

$$
L=\sqrt{\frac{R_{c} k_{w}}{L_{p}}},
$$

which gives a value of $0.779 \mathrm{~mm}$, which is reasonable for brain tissue which has a length of the order of millimetres. Similarly, the coefficient of the time derivative of pressures in eqn. 36 can also be set to one to give a characteristic time:

$$
T=\frac{R_{c}}{L_{p} Q_{w}}
$$

which gives a value of $51.4 \mathrm{~s}$, which is reasonable for a process that occurs over a timescale of minutes.

It can be noted from Table 1 that the ratio of the permeability of blood $k_{b}$ to the permeability of water $k_{w}$ is much larger than one:

$$
\frac{k_{b}}{k_{w}}=\frac{\kappa_{b} / \mu_{b}}{\kappa_{w} / \mu_{w}}>>1,
$$

implying that all coefficients in eqn. 35 with $k_{b}$ as their denominator are approximately zero and can be neglected, leaving only $\tilde{\nabla}^{2} \tilde{P}_{b}=0$, which means that blood pressure is effectively constant throughout space and equal to the baseline value of blood pressure, $\bar{P}_{b}$. Therefore, the system of equations can now be reduced to only two equations by removing all differential forms of $\bar{P}_{b}$, as shown below:

$$
\begin{gathered}
0=\tilde{\nabla}^{2} \tilde{u}-\frac{2}{\tilde{r}^{2}} \tilde{u}-\frac{\partial \tilde{P}_{w}}{\partial \tilde{r}} \\
\frac{\partial \tilde{P}_{w}}{\partial \tilde{t}}=\tilde{\nabla}^{2} \tilde{P}_{w}-2 \bar{n}_{b} f\left[\left(\tilde{P}_{b}-\tilde{P}_{w}\right)-\sigma \tilde{\Pi}_{b}\right] .
\end{gathered}
$$

The coefficient $\alpha_{w}$ can thus finally be included into a slightly revised relationship for characteristic length 37.

$$
L_{u}=\alpha_{w} \frac{\bar{P}(1-2 v)}{2 G(1-v)} L
$$

to give a revised value of $1.105 \mathrm{~mm}$. These equations can now be used to solve for tissue displacement and interstitial water pressure.

Lastly, referring back to eqn. 13 , non-dimensionalising the Péclet number yields:

$$
P e=\tilde{J}_{V A} \beta,
$$

where:

$$
\beta=\frac{\bar{J}_{V A}(1-\sigma) h}{D}
$$


and $\bar{J}_{V A}$ represents the characteristic water filtration flux, with a value of the order of $10^{-9} \mathrm{~m} / \mathrm{s}$. The capillary wall thickness, $h$ has a value of $0.5 \mu \mathrm{m}$, while the diffusion coefficient of protein, $D$ has a value of $5.62 \times 10^{-6} \mathrm{~m} 2 / \mathrm{s}\left[37\right.$. This gives a value of $\beta \approx 10^{-11}$, thus $P e<<1$.

\subsection{Model parameters and numerical procedures}

The shear modulus $G$ of cerebral tissue can be calculated from the value of tissue Young's modulus $E_{T}$ and Poisson's ratio $v$ from the relationship:

$$
G=\frac{E_{T}}{2(1+v)},
$$

The value of $E_{T}$ is not well known, thus is taken here from the case of hydrocephalic conditions, which are assumed to occur slowly and producing relatively larger $E_{T}$ compared to those found during neurosurgical processes, which produce smaller value of $E_{T}$ [28. Meanwhile, the value of $v$ is taken from the rough estimate of 38$]$.

There are four parameters that represent the characteristics of the fluid networks. They are the permeability $\kappa^{a}$, viscosity $\mu^{a}$, Biot parameter $\alpha^{a}$, and relative compressibility $Q^{a}$. The first three parameter values are taken directly from literature [26], where the parameter values were calculated through extensive parametric testing. Meanwhile the values of $Q^{a}$ are calculated from [31, 36, 35] eqn: 25. The tissue bulk modulus $K^{S}$ can be calculated from:

$$
K^{S}=\frac{2 G(1+v)}{3(1-2 v)} .
$$

The bulk modulus for blood is taken from [39], where the blood is assumed slightly compressible. Other parameters are taken directly from literature as shown in Table 1.

The system of eqns. 41 and 42 is used to solve for interstitial pressure and tissue displacement. The function $f$, which represents the fraction of vessels that remain open at each point in space and time, is approximated here using a sigmoid function:

$$
f=\frac{1}{2}\left(1+\tanh \left(\frac{1}{\epsilon}\left(1+\frac{\bar{P}-P_{w}}{E}\right)\right)\right)
$$

where the term $\epsilon=0.01$ is a scaling factor. This equation comes from setting the term in the bracket in eqn. 16 to be equal to zero. This is used to ensure that the value of $f$ can be either one (vessels open) or zero (vessels close), but that there is a small transition between these two states in order to ensure numerical stability.

The boundary conditions are set both at the origin (zero flux in all variables) and at a far boundary, where the variables return to the baseline values, as given:

$$
\left.\frac{\partial}{\partial \tilde{r}}\left(\tilde{u}, \tilde{P}_{w}\right)\right|_{\tilde{r}=0}=0 .
$$




$$
\begin{gathered}
\tilde{u}(\tilde{R}, \tilde{t}) \rightarrow 0, \\
\tilde{P}_{w}(\tilde{R}, \tilde{t}) \rightarrow 1 .
\end{gathered}
$$

Meanwhile, the initial conditions at $\tilde{t}=0$ are as shown:

$$
\begin{gathered}
\tilde{u}(\tilde{r}, 0)=0, \\
\tilde{P}_{w}(\tilde{r}, 0)=1 .
\end{gathered}
$$

where the initial pressure is assumed to be equal to its baseline value and the tissue initially has zero displacement. Thus, the initial temporal gradient of pressure is given by:

$$
\left.\frac{\partial \tilde{P}_{w}}{\partial \tilde{t}}\right|_{\tilde{t}=0}=\bar{n}_{b}\left[\left(\tilde{P}_{b}-\sigma \tilde{\Pi}_{b}\right)-1\right]
$$

A collapse front will exist at the points where the pressure is equal to a critical value given by:

$$
\tilde{P}_{c}=1+\tilde{E}
$$

Once pressure has reached this critical value, the differential equation reduces to:

$$
\frac{\partial \tilde{P}_{c}}{\partial \tilde{t}}=\tilde{\nabla}^{2} \tilde{P}_{c}
$$

which is a linear diffusion equation. After pressure has reached its critical value, there is no further water leakage from the collapse vessels. The collapse process is assumed here to be irreversible.

Typical adult brain volume is about 1130 to $1260 \mathrm{~cm}^{3}$ [40], implying that the skull radius is somewhere around 65 to $67 \mathrm{~mm}$, assuming that the brain is a perfect sphere. Hence, a maximum length range of $\tilde{R}=100$ is chosen, representing the solid boundary of the skull. The equations are solved using a standard non-linear parabolic-elliptic partial differential equation (PDE) solver in MATLAB.

\section{Results}

For all of the results, the core zone, which is the region of BBB breakdown, is initially set to be of radius 4 length units (LU) $(3.116 \mathrm{~mm})$, unless otherwise stated. This value represents a typical medium sized infarct. Figure 3 shows the changes in tissue displacement and interstitial pressure with time and radius. The water pressure initially increases sharply and reaches the maximum pressure at about 2200 time units (TU) (1.3 days) after constant reperfusion. The maximum pressure is 1.198 pressure units (PU) (11.98 mmHg), which is below the critical pressure (1.65 PU or $16.5 \mathrm{mmHg}$ ). This indicates that the vessels do not collapse under these conditions. Meanwhile, the tissue displacement increases slowly compared to the rise in the water 
Table 1: List of parameters and their baseline value for the proposed model.

\begin{tabular}{|c|c|c|}
\hline Parameter & Value & Reference(s) \\
\hline Poisson's ratio $v$ & 0.35 & 38 . \\
\hline Shear modulus $G$ & $216.3 \mathrm{~Pa}$ & $\begin{array}{l}\text { Calculated using } E_{T}=584 \mathrm{~Pa} \\
\text { [28] and eqn. } 46\end{array}$ \\
\hline Permeability of blood, $\kappa_{b}$ & $1.0 \times 10^{-10} \mathrm{~m}^{2}$ & $26]$ \\
\hline Viscosity of blood, $\mu_{b}$ & $2.67 \times 10^{-3} \mathrm{~Pa} \cdot \mathrm{s}$ & $26]$. \\
\hline Biot parameter for blood network, $\alpha_{b}$ & 1 & [26]. \\
\hline Relative compressibility of blood, $Q_{b}$ & $669.0 \mathrm{~Pa}$ & $\begin{array}{l}\text { Using eqn. } 2547 \text { and } K_{b}= \\
1.0 \times 10^{10} \mathrm{~Pa} 39 \text {. }\end{array}$ \\
\hline Permeability of water, $\kappa_{w}$ & $3.6 \times 10^{-15} \mathrm{~m}^{2}$ & 21 . \\
\hline Viscosity of water, $\mu_{w}$ & $1.0 \times 10^{-3} \mathrm{~Pa} \cdot \mathrm{s}$ & Value for water at $20^{\circ} \mathrm{C}$. \\
\hline Biot parameter for water network, $\alpha_{w}$ & 1 & $26]$. \\
\hline Relative compressibility of water, $Q_{w}$ & $3244 \mathrm{~Pa}$ & $\begin{array}{l}\text { Using eqn. } 2548, n_{w}=0.8 \text { and } \\
K_{w}=2.2 \times 10^{9} \mathrm{~Pa} \text {. }\end{array}$ \\
\hline Hydraulic permeability, $L_{p}$ & $\begin{array}{l}2.9 \times 10^{-9} \mathrm{~m} / \mathrm{s} \cdot \mathrm{cmH} 2 \mathrm{O} \text { or } \\
3.0 \times 10^{-11} \mathrm{~m} / \mathrm{s} \cdot \mathrm{Pa}\end{array}$ & 21. \\
\hline Reflection coefficient, $\sigma$ & 0.93 & 21 \\
\hline Capillary osmotic pressure, $\Pi_{b}$ & $2445 \mathrm{~Pa}$ or $25 \mathrm{cmH} 2 \mathrm{O}$ & 21 \\
\hline Blood pressure, $\bar{P}_{b}$ & $4389 \mathrm{~Pa}$ or $33 \mathrm{mmHg}$ & 41 \\
\hline Baseline value of pressure, $\bar{P}$ & $1330 \mathrm{~Pa}$ or $10 \mathrm{mmHg}$ & 42 \\
\hline Typical capillary radius, $R_{c}$ & $5 \times 10^{-6} \mathrm{~m}$ & 43 \\
\hline Capillary wall stiffness, $E$ & $864.5 \mathrm{~Pa}$ or $6.5 \mathrm{mmHg}$ & 44 \\
\hline Baseline value of blood volume fraction, $\bar{n}_{b}$ & 0.03 & 45 \\
\hline
\end{tabular}

pressure. The maximum tissue displacement is 0.240 displacement-length units (DLU) (0.265 mm), which occurs at distance $8.8 \mathrm{LU}(6.69 \mathrm{~mm})$ at time $5000 \mathrm{TU}$. This suggests that the tissue spreads as the interstitial pressure increases due to water filtration, but that this occurs slowly.

When the core zone radius is varied while other parameter values are set at baseline conditions, the results shown in figure 3 are obtained. The pressure reaches a maximum value at $1.58 \mathrm{PU}(15.80 \mathrm{mmHg})$, which is slightly below the critical value $(16.50 \mathrm{mmHg})$, indicating that vessels will never collapse at the baseline conditions. The normal value of ICP is in the range of 7 to $15 \mathrm{mmHg}$ [46]. However, ICP values of more than $20 \mathrm{mmHg}$ should be monitored as high ICP increases the chances of mortality [47. On the other hand, the tissue displacement increases, reaching a maximum value at about $7.5 \mathrm{DLU}$ (8.288 mm) at core radius of about $50 \mathrm{LU}(38.95 \mathrm{~mm})$. The position of maximum displacement increases sharply at a core 
(a)

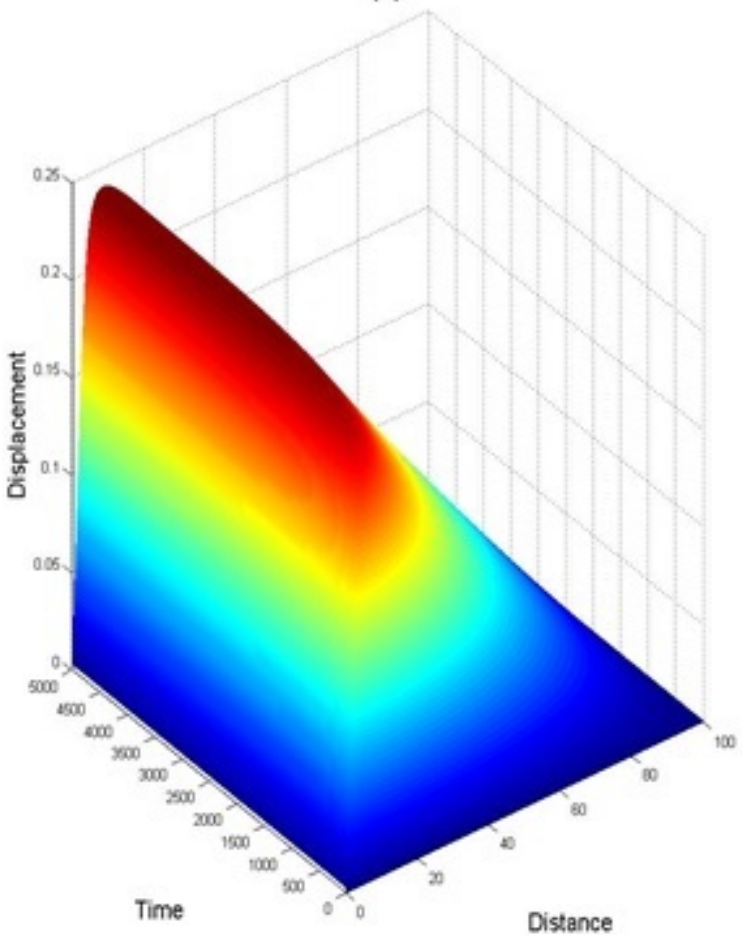

(b)

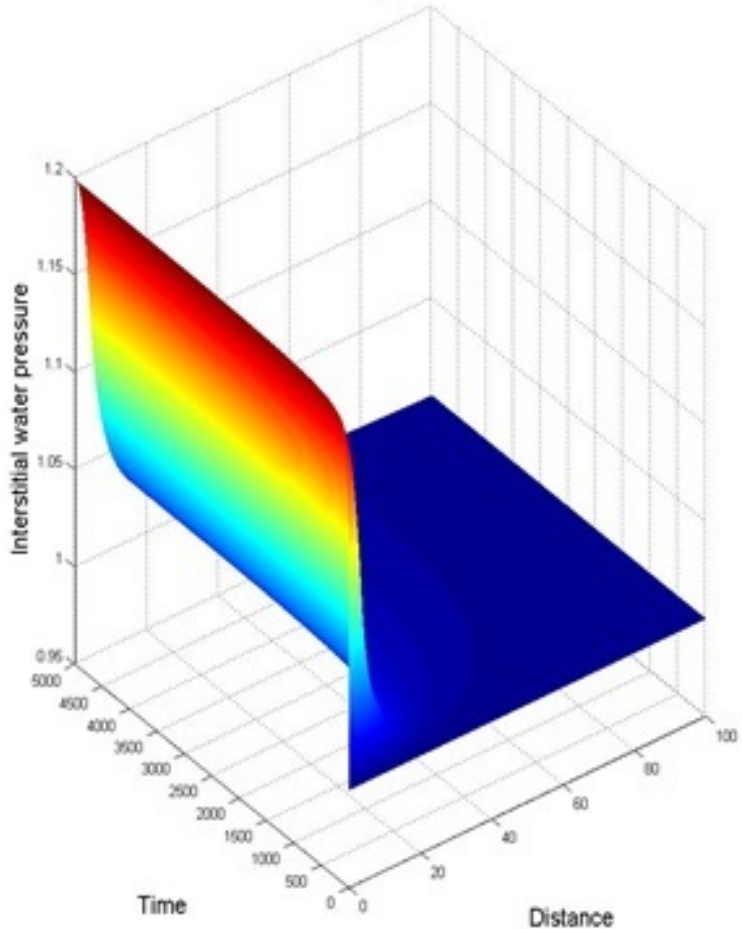

Figure 3: Time courses of vessel behaviour using baseline parameters values: (a) tissue displacement; (b) interstitial water pressure.

radius less than $5 \mathrm{LU}(3.895 \mathrm{~mm})$ but then increases almost linearly, reaching a maximum value of about 53 LU $(41.29 \mathrm{~mm})$.

There are other parameters that will affect the behaviour of the model, the most important of which are the capillary stiffness $E$, blood pressure $P_{b}$, reflection coefficient $\sigma$ and capillary osmotic pressure $\Pi$. The driving pressure, given by eqn. 54 must be positive to allow capillary filtration to occur. Hence, the ranges of blood pressure and osmotic pressure that will cause water flux into tissue space are:

$$
\begin{gathered}
P_{b}>27.10 \mathrm{mmHg}, \\
\Pi_{b}<33.63 \mathrm{cmH} 2 \mathrm{O},
\end{gathered}
$$

when assuming baseline values for other variables.

Figure 5 shows the change in maximum displacement and its position and maximum pressure against time when $P_{b}$ is varied. Maximum water pressure increases, reaching a plateau when $P_{b}$ increases. Collapse occurs at $P_{b}=50$ and $60 \mathrm{mmHg}$ as shown in figure 5(b). For $P_{b}=50 \mathrm{mmHg}$, the rise in water pressure to reach the critical value is slow, about $41 \mathrm{TU}$ (0.6 hours) after reperfusion. Meanwhile, for $P_{b}=60 \mathrm{mmHg}$, the rise in water pressure is quick, taking about $6.5 \mathrm{TU}$ (5.6 minutes) to reach the critical pressure. The higher the reperfusion pressure, the faster the vessel collapse occurs. Furthermore, the maximum displacement also 
(a)

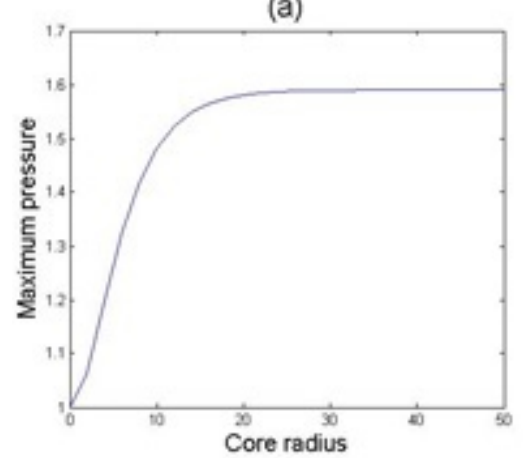

(b)

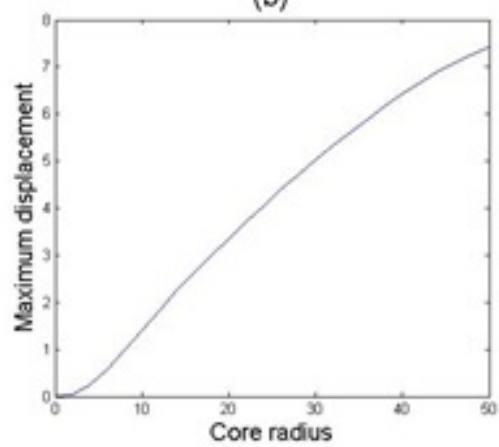

(c)

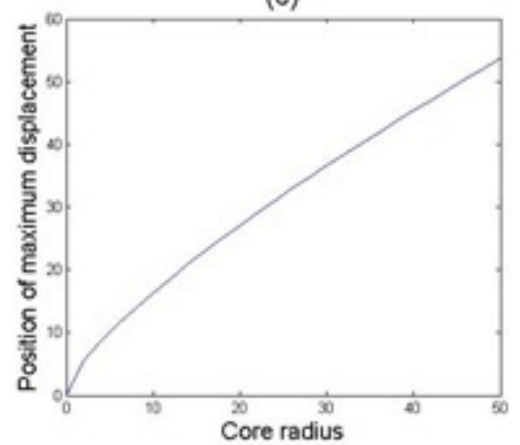

Figure 4: Vessel behaviour using baseline parameters values with varying core radius: (a) maximum pressure; (b) maximum displacement; (c) position of maximum displacement.

increases when $P_{b}$ increases and reaches a maximum value of $0.99 \mathrm{DLU}(1.094 \mathrm{~mm})$ at $P_{b}=60 \mathrm{mmHg}$ with the position of maximum displacement found at $9.26 \mathrm{LU}(7.214 \mathrm{~mm})$. Tissue is displaced as $P_{b}$ increases to give space for the amount of water flux into the interstitial space. For other values of $P_{b}$ that do not cause collapse, the position of maximum displacement shows similar values and trends. As $P_{b}$ increases and the vessels start to collapse, the position of maximum displacement also starts to increase.

(a)

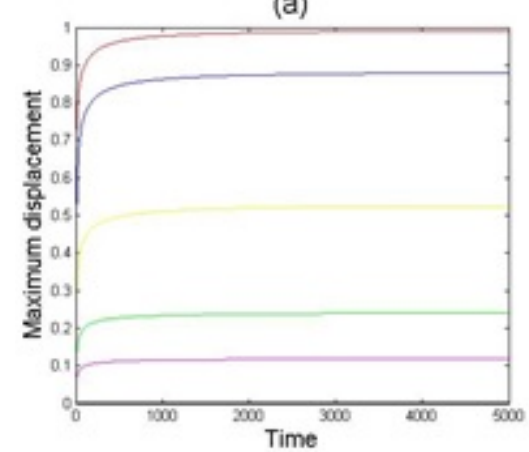

(b)

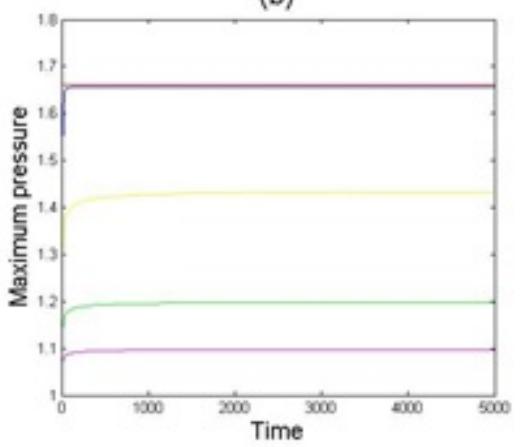

(c)

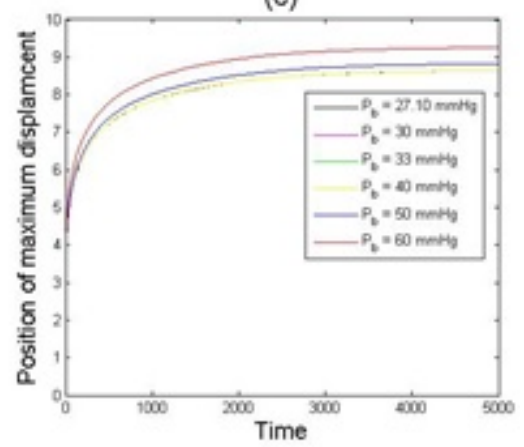

Figure 5: Time courses of vessel behaviour using baseline values with varying $P_{b}$ : (a) maximum displacement; (b) maximum pressure; (c) position of maximum displacement.

Figure 6 shows the results obtained when is varied while other parameters are kept at baseline values. The maximum displacement and maximum pressure both increase as decreases. When $\sigma=0$, vessels collapse occurs and the time of collapse occurrence is about $39.3 \mathrm{TU}$ (0.56 hours) after reperfusion. Zero $\sigma$ means all protein plasma can filtrate through the capillary BBB and this decreases the osmotic pressure inside the interstitial space, which allows more water to flow into it. The maximum tissue displacement when $\sigma=0$ is $0.8805 \mathrm{DLU}(0.9730 \mathrm{~mm})$ which occurs at $4.83 \mathrm{LU}(3.763 \mathrm{~mm})$ from the core radius. For the other values of $\sigma$, the position of maximum displacement is found to be roughly the same. Another interesting result is that at $\sigma=1$, where there is a total blockage of protein plasma into the interstitial space, there is still an increase in tissue displacement and water pressure. This is due to the difference in blood and water 
pressures that causes water to filtrate into the interstitial space. However, a balance between too high and too low a value of reperfusion pressure is important to avoid the risk of vessel collapse.

(a)

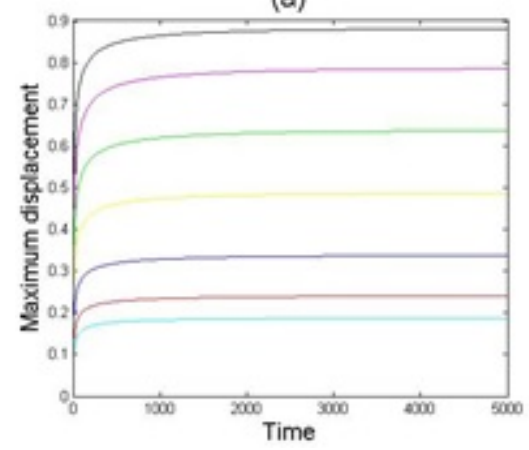

(b)

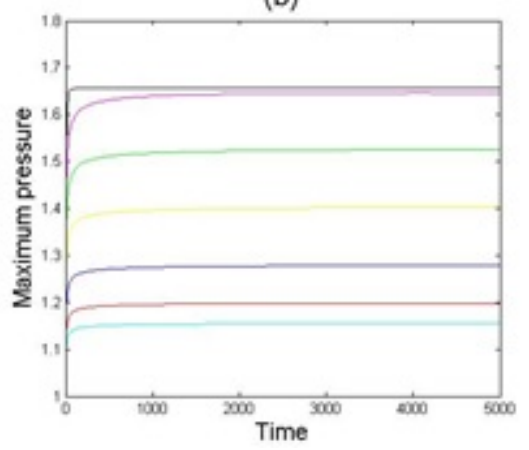

(c)

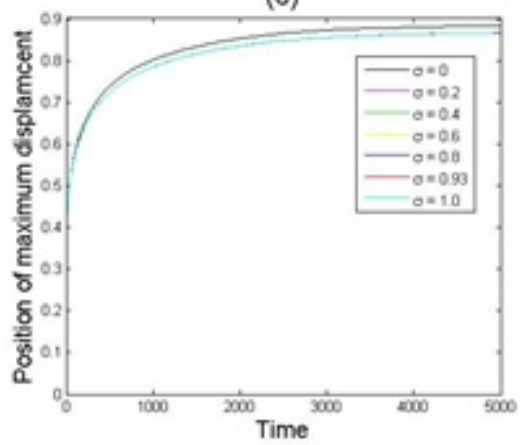

Figure 6: Time course of vessel behaviour using baseline values with varying $\sigma$ : (a) maximum displacement; (b) maximum pressure; (c) position of maximum displacement.

The results for varying $\Pi$ are as shown in figure 6 . The maximum displacement and maximum pressure both increase when $\Pi$ decreases. Vessel collapse occurs when $\Pi=0$, showing that water filtration occurs due to the difference in blood and water pressures only. The occurrence of vessel collapse is about $39.3 \mathrm{TU}$ (0.56 hours) after reperfusion, similar to the results when $\sigma=0$. The maximum displacement during collapse is the same as when $\sigma=0$, i.e. $0.8805 \mathrm{DLU}(0.9730 \mathrm{~mm})$. The position of maximum displacement when $\Pi=0$ is at $8.83 \mathrm{LU}(6.879 \mathrm{~mm})$, meanwhile for other values of $\Pi$, the position of maximum displacement is the same. Giving reperfusion containing a high concentration of protein plasma can provide a high $\Pi$ value, but too high a value of $\Pi$ can also cause vessel collapse.

(a)

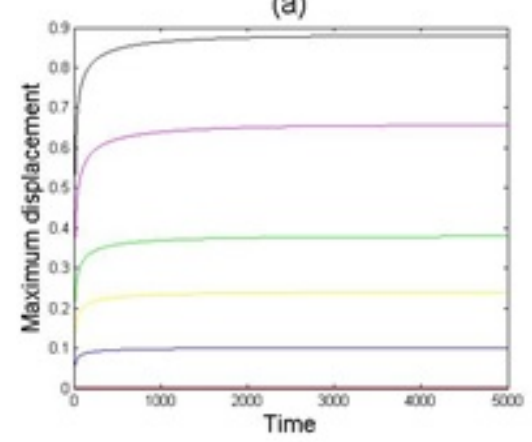

(b)

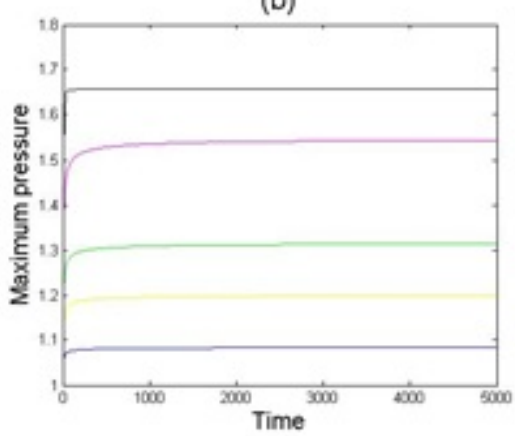

(c)

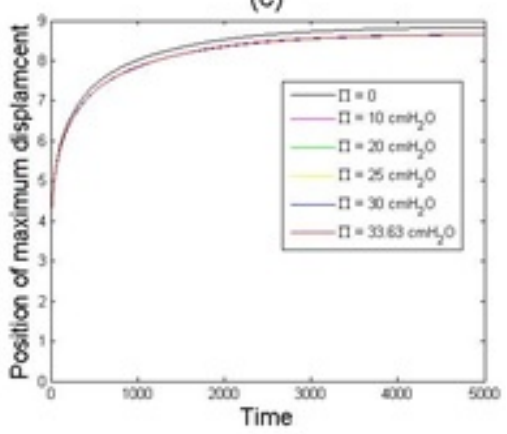

Figure 7: Time course of vessel behaviour using baseline values with varying П: (a) maximum displacement; (b) maximum pressure; (c) position of maximum displacement.

Figure 8 shows the critical pressure-capillary stiffness relationship obtained when $E$ is varied while other parameters are kept at baseline conditions. Collapse will occur when water pressure reaches the critical value given by eqn. 56. In figure 8, the minimum $E$ before collapse occurs is estimated to be 0.1983 $(\tilde{E}=1.1983-1) \mathrm{PU}(263.74 \mathrm{~Pa})$. Any capillaries with stiffness higher than this may have less chance to 
collapse. The maximum value of $P_{c}$ reached when $E$ is varied is $1.1993 \mathrm{PU}(1595 \mathrm{~Pa})$ and this remains constant for higher $E$ values. From this result, it shows that having low capillary stiffness can cause vessel collapse.

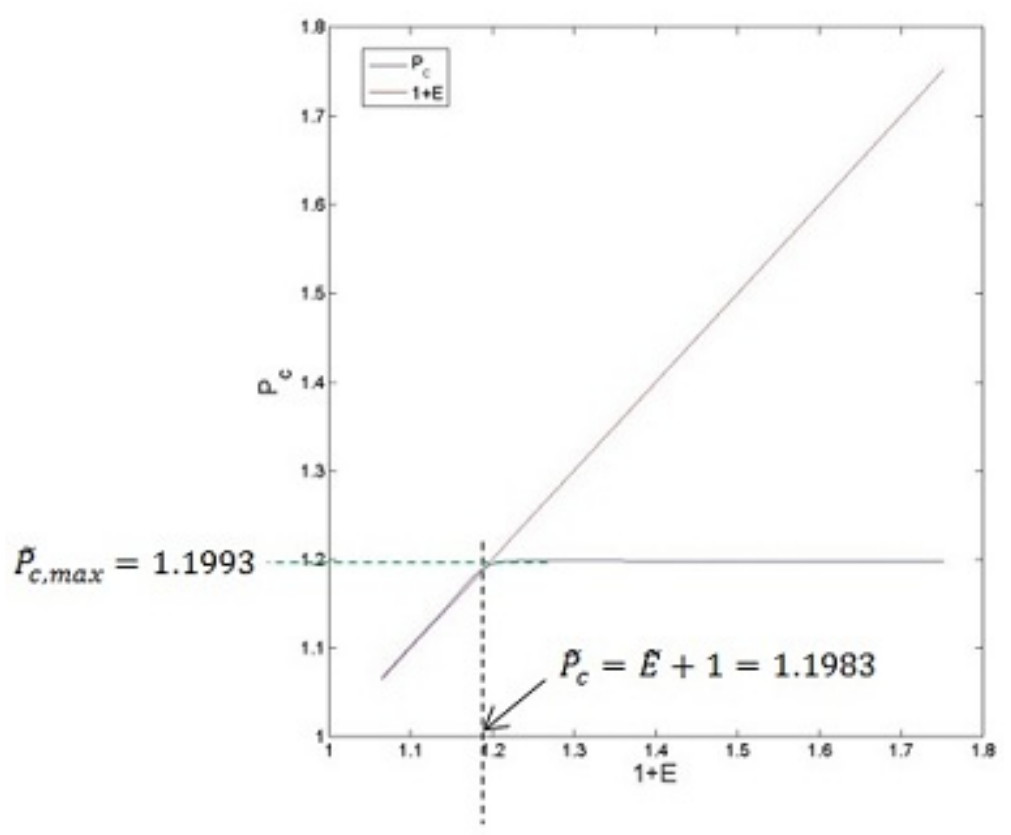

Figure 8: Relationship between critical pressure and capillary stiffness. The terms $P_{c}$ and $E$ shown in the figure represent the non-dimensionalized critical pressure and capillary stiffness.

\section{Discussion}

The model proposed here has been used to investigate the possibility of vessel collapse when reperfusion is carried out for patients after ischemic stroke. Under normal conditions, a low value of hydraulic permeability of the normal BBB and $\mathrm{CBF}$ autoregulation both maintain water flow into the interstitial space and hence regulate brain volume 48. Hence, assuming that there is no net water flux through the BBB is reasonable because the water flow is tightly regulated. After an ischemic attack, BBB breakdown can occur and its permeability increases. The hydraulic permeability of the pathological BBB was estimated here to be $29 \times 10^{-9} \mathrm{~m} / \mathrm{s} . \mathrm{cmH} 2 \mathrm{O}$, which was taken from the study done by [21], that based on the results of [11] and 23. Meanwhile, the reflection coefficient in the model is taken specifically for protein plasma. It is assumed that the value of for ions is zero, which means that all ions can freely enter the interstitial space when BBB breakdown occurs. Both of the $\sigma$ values are taken to be the same as the properties of peripheral capillaries because when the BBB breaks down, its properties are assumed to be the same as those in peripheral capillaries [21].

An increase in BBB permeability after ischemic attack causes water flux into the cerebral tissue space when reperfusion is applied. This is shown by the results where the interstitial pressure increases when 
the reperfusion pressure increases. This is also shown by cerebral tissue displacement, which reflects the occurrence of tissue swelling. The increase in both the tissue interstitial pressure and displacement can cause capillaries to collapse. From the results shown here, it is found that vessel collapse occurs at high value of reperfusion pressure, low value of blood osmotic pressure, low value of BBB reflection coefficient and also at low value of capillary stiffness.

It should be noted here that collapse is assumed to occur when the term $f$ becomes zero. This term is an approximation of the integration in eqn. 4. assuming that all vessels have the same dimensions and mechanical properties. To investigate the effect of different vessels properties would require setting up a full integral for that equation. The effect of BBB breakdown on the mechanical properties of cerebral capillaries may also affect the collapse occurrence. There are very limited data available for cerebral capillary stiffness and hence, an approximation is made by taking the capillary stiffness value from [44], which, it should be noted, was a study on tumour microcirculation.

The model proposed here does not consider the contribution of local cerebral autoregulation, which may have an effect on controlling transmural pressure. Generally, cerebral autoregulation impairment occurs after stroke and any recovery may take up to 3 months [49. Impairment of local autoregulation in the infarcted region will likely intensify the reperfusion injury [50. However, it is still not very clear whether there is disruption to local cerebral autoregulation after ischemic stroke [51. Including the dynamics of cerebral autoregulation would provide a better understanding of vessel collapse occurrence.

An assumption of the homogeneity of cerebral tissue is made here for the purpose of model simplification. In reality, the white matter possesses a higher tissue compliance than grey matter, which allows it to easily expand when water filtrates [52]. Vasogenic oedema occurs most commonly in the white matter and this is due to structural differences, where white matter is more homogeneous whilst grey matter is more twisted [53. In the study done by [54, it was found that swelling of the white matter due to vasogenic oedema is anisotropic, which depends on the nerve fibres orientation [55]. Incorporation of the anisotropy of white matter into the current model would be a worthwhile avenue for further investigation [24, 56, [57].

Another aspect worth studying in future is the vasogenic oedema resolution. The oedematous fluid will move out into the ventricles and subarachnoid spaces via glia limitans, into the capillary endothelium via the astrocytic foot or by metabolic degradation [58, 59] The presence of aquaporin-4 (AQP4) channels in the glia limitans and astrocytic foot [59] facilitate the removal of the oedematous fluid. However, AQP4 also plays a role in the formation of cytotoxic oedema that causes intracellular swelling, which does not result in an increase in ICP and brain tissue swelling [15. Modification of the current model through inclusion of the role of AQP4 might provide new insights into the occurrence of reperfusion injury and its prevention.

It should be noted that there are other causes of the no-reflow phenomenon instead of vessel compression, which includes elevated blood viscosity [60, oxidative stress [61, 62, leukocyte adhesion [63, 64] and postischemic hypotension [65]. During ischemia, erythrocytes and platelets accumulate in the cerebral vessels, which will impede cerebral blood flow 65. In addition, restoration of oxygen after ischemia may create 
oxygen free radicals and initiate complex cascade activations of metalloproteinase (MMP) that will cause irreversible damage to the cerebral vessel endothelium [62. In a study done by [66], within the first 4 hours of middle cerebral artery occlusion (MCAO), leukocytes were found to adhere to the cerebral vessels endothelium, which may also block the blood flow.

\section{Conclusion}

In this paper, we have proposed a new model to study ischemia-reperfusion injury by exploring the possibility of vessel collapse through blood-brain barrier breakdown and water filtration. The resulting increase in interstitial pressure and the tissue swelling have been hypothesized to cause vessel collapse. The results presented here show that vessel collapse can indeed occur, but only at high values of reperfusion pressure, blood osmotic pressure, cerebral capillary permeability and a low value of cerebral capillary stiffness.

Although the no-reflow phenomenon is clearly a complex, multi-factorial effect, we have shown here that under certain conditions vessel collapse can indeed play a role in this phenomenon. Such behaviour will also very likely be influenced by and influence other physiological factors and processes. It remains to be seen how the different components involved in this effect interact, to understand how it behaves more fully and thus to help to prevent its recurrence in clinical practice.

\section{References}

[1] World Health Organization, The top 10 causes of death. july 2013 (2014).

[2] V. L. Feigin, C. M. M. Lawes, D. A. Bennett, S. L. Barker-Collo, V. Parag, Worldwide stroke incidence and early case fatality reported in 56 population-based studies: A systematic review, Lancet Neurol. 8 (2009) 355-369.

[3] Royal College of Physicians, National sentinel stroke clinical audit 2010 round 7: Public report for england, wales and northern ireland. prepared on behalf of the intercollegiate stroke working party may 2011, Tech. rep. (2011).

[4] A. J. Furlan, I. L. Katzan, L. R. Caplan, Thrombolytic therapy in acute ischemic stroke, Curr. Treat. Options Cardiovasc. Med. 5 (2003) 171-180.

[5] W. S. Smith, G. Sung, S. Starkman, J. L. Saver, C. S. Kidwell, Y. P. Gobin, H. L. Lutsep, G. M. Nesbit, T. Grobelny, M. M. Rymer, I. E. Silverman, R. T. Higashida, R. F. Budzik, M. P. Marks, Safety and efficacy of mechanical embolectomy in acute ischemic stroke, Stroke 36 (2005) 1432-1438.

[6] E. C. Haley Jr, T. G. Brott, G. L. Sheppard, W. Barsan, J. Broderick, J. R. Marler, G. L. Kongable, J. Spilker, S. Massey, C. A. Hansen, Pilot randomized trial of tissue plasminogen activator in acute ischemic stroke: The tpa bridging study group, Stroke 24 (1993) 1000-1004. 
[7] M. G. Lansberg, E. Bluhmki, V. N. Thijs, Efficacy and safety of tissue plasminogen activator 3 to 4.5 hours after acute ischemic stroke: A metaanalysis, Stroke 40 (2009) 2438-2441.

[8] A. Ames, R. L. Wright, M. Kowada, J. M. Thurston, G. Majno, Cerebral ischemia: Ii. the no-reflow phenomenon, Am. J. Pathol. 52 (1968) 437-453.

[9] K. M. Mohan, C. D. A. Wolfe, A. G. Rudd, P. U. Heuschmann, P. L. Kolominsky-Rabas, A. P. Grieve, Risk and cumulative risk of stroke recurrence: A systematic and meta-analysis, Stroke 42 (2011) 14891494.

[10] N. J. Abbott, L. Ronnback, E. Hansson, Astrocyte-endotehlial interactions at the blood-brain barrier, Nat. Rev. Neurosci. 7 (2006) 41-53.

[11] G. Li, W. Yuan, B. M. Fu, A model for the blood-brain barrier permeability to water and small solutes, J. Biomech 43 (2010) 2133-2140.

[12] M. Krueger, I. Bechmann, Cns pericytes: Concepts, misconceptions, and a way out, Glia 58 (2009) $1-10$.

[13] J. Greenwood, Mechanisms of blood-brain barrier breakdown, Neuroradiology 33 (1991) 95-100.

[14] A. W. Unterberg, J. Stover, B. Kress, K. L. Kiening, Edema and brain trauma, Neuroscience 129 (2004) $1021-1029$.

[15] J. J. Donkin, R. Vink, Mechanisms of cerebral edema in traumatic brain injury: Therapeutic developments, Curr. Opin. Neurol. 23 (2010) 293-299.

[16] G. J. del Zoppo, Microvascular changes during cerebral ischemia and reperfusion, Cerebrovasc. Brain Metab. Rev. 6 (1994) 47-96.

[17] E. H. Starling, On the absorption of fluids from the connective tissue spaces, J. Physiol. 19 (4) (1896) $312-326$.

[18] J. R. Levick, C. C. Michel, Microvascular fluid exchange and the revised starling principle, Cardiovasc. Res. 87 (2010) 198-210.

[19] B. Rippe, B. Haraldsson, Transport of macromolecules across microvascular walls: The two-pore theory, Physiol. Rev. 74 (1) (1994) 163-219.

[20] E. Preston, D. O. Foster, Evidence for pore-like opening of the blood-brain barrier following forebrain ischemia in rats, Brain Res. 761 (1997) 4-10.

[21] S. W. Su, Modelling blood flow and oxygen transport in the human cerebral cortex, Thesis (2011). 
[22] P. A. Fraser, A. D. Dallas, Measurement of filtration coefficient in single cerebral microvessels of the frog, J. Physiol. 423 (1990) 343-361.

[23] Y. Hakamata, U. Ito, S. Hanyu, M. Yoshida, Long-term high-colloid oncotic therapy for ischemic brain edema in gerbils, Stroke 26 (1995) 2149-2158.

[24] P. J. Basser, Interstitial pressure, volume, and flow during infusion into brain tissue, Microvasc. Res. 44 (1992) 143-165.

[25] S. K. Kyriacou, A. Mohamed, K. Miller, S. Neff, Brain mechanics for neurosurgery: Modeling issues, Biomechan. Model. Mechanobiol. 1 (2002) 151-164.

[26] B. Tully, Y. Ventikos, Cerebral water transport using multiple-network poroelastic theory: Application to normal pressure hydrocephalus, J. Fluid Mech. 667 (2011) 188-215.

[27] I. Sobey, B. Wirth, Effect of non-linear permeability in a spherically symmetric model of hydrocephalus, Math. Med. Biol. 23 (2006) 339-361.

[28] Z. Taylor, K. Miller, Reassessment of brain elasticity for analysis of biomechanisms of hydrocephalus, J. Biomech. 37 (2004) 1263-1269.

[29] K. Miller, Constitutive model of brain tissue suitable for finite element analysis of surgical procedures, J. Biomech. 32 (1999) 531-537.

[30] M. I. Miga, K. D. Paulsen, P. J. Hoopes, J. Francis E. Kennedy, A. Hartov, D. W. Roberts, In vivo quantification of a homogeneous brain deformation model for updating preoperative images during surgery, IEEE T. Biomed. Eng. 47 (2) (2000) 266-273.

[31] M. Bai, Multiporosity/multipermeability approach to the simulation of naturally fractured reservoirs, Water Resour. Res. 29 (6) (1993) 1621-1633.

[32] M. A. Biot, Mechanics of deformation and acoustic propagation in porous media, J. Appl. Phys. 33 (4) (1962) 1482-1498.

[33] K. Terzaghi, Theoretical soil mechanics, John Wiley, New York, 1943.

[34] M. A. Biot, D. G. Willis, The elastic coefficients of the theory of consolidaton, J. Appl. Mech. 24 (1957) 594-601.

[35] O. C. Zienkiewicz, Dynamic behaviour of saturated porous media: The generalized biot formulation and its numerical solution, Int. J. Numer. Anal. Met. 8 (1984) 71-96.

[36] O. C. Zienkiewicz, A. H. C. Chan, M. Pastor, D. K. Paul, T. Shiomi, Static and dynamic behaviour of soils: A rational approach to quantitative solutions. i. fully saturated problems, Proc. R. Soc. Lond. A. 429 (1990) 285-309. 
[37] X. Zhang, R. H. Adamson, F.-R. E. Curry, S. Weinbaum, A 1d model to explore the effects of tissue loading and tissue concentration gradients in the revised starling principle, Am. J. Physiol. Heart Circ. Physiol. 291 (2006) H2950-H2964.

[38] J. M. Drake, O. Mostachfi, G. Tenti, S. Sivaloganathan, Realistic simple mathematical model of brain biomechanics for computer simulation of hydrocephalus and other brain abnormalities, Can. J. Neurol. Sci. 23 (1996) S5.

[39] H. Masugata, S. Senda, K. Mizushige, X. Lu, A. Kinoshita, H. Sakamoto, S. Nozaki, S. Sakamoto, H. Matsuo, Mitral valve tissue characterization using acoustic microscopy, J. Cardiol. 31 (Suppl. 1) (1998) $45-51$.

[40] K. P. Cosgrove, Evolving knowledge of sex differences in brain structure, function and chemistry, Biol. Psychiat. 62 (8) (2007) 847-855.

[41] C. Lucas, An anatomical model of the cerebral vasculature and blood flow, Ph.D. thesis (2013).

[42] S. J. Payne, A model of the interaction between autoregulation and neural activation in the brain, Mathematical Biosciences 204 (2) (2006) 260-281.

[43] F. Cassot, F. Lauwers, C. Fouard, S. Prohaska, V. Lauwers-Cances, A novel three-dimensional computerassisted method for a quantitative study of microvascular networks of the human cerebral cortex, Microcirculation 13 (2006) 1-18.

[44] J. W. Baish, P. A. Netti, R. K. Jain, Transmural coupling of fluid flow in microcirculatory network and interstitium in tumors, Microvasc. Res. 53 (1997) 128-141.

[45] H. Ito, I. Kanno, H. Iida, J. Hatazawa, E. Shimosegawa, H. Tamura, T. Okudera, Arterial fraction of cerebral blood volume in humans measured by positron emission tomography, Ann. Nucl. Med. 15 (2) (2001) 111-116.

[46] L. A. Steiner, P. J. D. Andrews, Monitoring the injured brain: Icp and cbf, Brit. J. Anaesth. 97 (1) (2006) 26-38.

[47] M. Balestreri, M. Czosnyka, P. Hutchinson, L. A. Steiner, M. Hiler, P. Smielewski, J. D. Pickard, Impact of intracranial pressure and cerebral perfusion pressure on severe disability and mortality after head injury, Neurocrit. Care 4 (2006) 8-13.

[48] T. Doczi, Volume regulation of the brain tissue - a survey, Acta Neurochir. (Wien) 121 (1993) 1-8.

[49] M. J. H. Aries, J. W. Elting, J. D. Keyser, B. P. H. Kremer, P. C. A. J. Vroomen, Cerebral autoregulation in stroke: A review of transcranial doppler studies, Stroke 41 (2010) 2697-2704. 
[50] M. Reinhard, S. Rutsch, J. Lambeck, C. Wihler, M. Czosnyka, C. Weiller, A. Hetzel, Dynamic cerebral autoregulation associates with infarct size and outcome after ischemic stroke, Acta Neurol. Scand. 125 (2012) 156-162.

[51] J. Dedrick Jordan, W. J. Powers, Cerebral autoregulation and acute ischemic stroke, Am. J. Hypertens. 25 (9) (2012) 946-950.

[52] H. K. Kimelberg, Water homeostasis in the brain: Basic concepts, Neuroscience 129 (2004) 851-860.

[53] I. Klatzo, Pathophysiological aspects of brain edema, Acta Neuropathol. 72 (1987) 236-239.

[54] T. Kuroiwa, M. Ueki, Q. Chen, S. Ichinose, R. Okeda, Is the swelling in brain edema isotropic or anisotropic?, Acta Neurochir. Suppl. (Wien) 60 (1994) 155-157.

[55] T. Kuroiwa, J. Yokofujita, H. Kaneko, R. Okeda, Accumulation of oedema fluid in deep white matter after cerebral cold injury, Acta Neurochir. Suppl. (Wien) 51 (1990) 84-86.

[56] K. H. Stverud, M. Darcis, R. Helmig, S. M. Hassanizadeh, Modeling concentration distribution and deformation during convection-enhanced drug delivery into brain tissue, Transp. Porous. Med. 92 (2012) $119-143$.

[57] K. Shahim, J. M. Drezet, J. F. Molinari, R. Sinkus, S. Momjian, Finite element analysis of normal pressure hydrocephalus: Influence of csf content and anisotropy in permeability, Appl. Bionics Biomech. 7 (3) (2010) 187-197.

[58] T. Tourdias, N. Mori, I. Dragonu, N. Cassagno, C. Boiziau, J. Aussudre, B. Brochet, C. Moonen, K. G. Petry, V. Dousset, Differential aquaporin 4 expression during edema build-up and resolution phases of brain inflammation, J. Neuroinflamm. 8 (2011) 143-160.

[59] M. C. Papadopoulos, G. T. Manley, S. Krishna, A. S. Verkman, Aquaporin-4 facilitates reabsorption of excess fluid in vasogenic brain edema, FASEB J. 18 (1291-1293).

[60] E. G. Fischer, A. Ames, A. V. Lorenzo, Cerebral blood flow immediately following brief circulatory stasis, Stroke 10 (1979) 423-427.

[61] T. Aoki, T. Sumii, T. Mori, X. Wang, E. H. Lo, Blood-brain barrier disruption and matrix metalloproteinase-9 expression during reperfusion injury: Mechanical versus embolic focal ischaemia in spontaneously hypertensive rats, Stroke 33 (2002) 2711-2717.

[62] Y. Yang, G. A. Rosenberg, Blood-brain barrier breakdown in acute and chronic cerebrovascular disease, Stroke 42 (2011) 3323-3328.

[63] G. J. del Zoppo, T. Mabuchi, Cerebral microvessel responses to focal ischemia, J. Cereb. Blood Flow Metab. 23 (2003) 879-894. 
[64] J. Huang, U. M. Upadhyay, R. J. Tamargo, Inflammation in stroke and focal ischemia, Surg. Neurol. 66 (2006) 232-245.

[65] K. A. Hossmann, Reperfusion of the brain after global ischemia: Hemodynamic disturbances, Shock 8 (2) (1997) 95-101.

[66] G. J. del Zoppo, G. W. Schmid-Schonbein, E. Mori, Polymorphonuclear leukocytes occlude capillaries following middle cerebral artery occlusion and reperfusion in baboons, Stroke 22 (1991) 1276-1283. 